\title{
SHORT-PULSED LASER WRITING IN MAGNETO-OPTICAL RECORDING
}

\author{
Hidehiko Wakabayashi and Fumiaki Yamada \\ IB.II Research, Tokyo Research Laboratory, \\ 5-19 Sanban-cho, Chiyoda-ku, Tokyo 102, Japan
}

Abstract - It was demonstrated that a writing process starts to approach an adiabatic condition when the duration of laser irradiation is reduced to about $10 \mathrm{~ns}$. The resultant steep temperature profile and reduction in the level of energy necessary for writing were found to be advantageous for writing small marks and for suppressing thermal interference effect.

KEYTVORDS: MAGNETO-OPTICAL RECORDING, SHORT PULSE, WRITING PROCESS, TEMPERATURE PROFILE, THERMAL INTERFERENCE

\section{INTRODUCTION}

In addition to the effort of reducing the laser wavelength, various techniques have been employed to achieve higher density in magneto-optical (MO) recording. Mark-edge recording methods with equalizations and super resolution techniques have been intensively studied to improve the readout process. However, accurate control of the mark edge and stable generation of small marks in the writing process are prerequisite for these techniques. The field modulation method has advantages in producing small marks and in mark edge control, but its application to drives with very high data rates is not yet well established. Various types of write compensation techniques have been proposed for light-intensity-modulation mark-edge recording to avoid thermal interaction within a (long) mark and between closely neighboring marks.

In the current generation MO drives, laser irradiation with a duration of more than $30 \mathrm{~ns}$ and a peak power of less than $10 \mathrm{~mW}$ is normally used for writing. In such a writing process, a considerable fraction of the injected energy is thought to be lost as a result of thermal diffusion during the irradiation, which in turn becomes the source of a thermal interaction effect. The idea of short-pulsed laser writing is to use laser irradiation of shorter duration and with higher peak power than in the conventional writing process, so that the injection of energy necessary for the formation of marks is completed before substantial thermal diffusion takes place. As the writing process becomes more adiabatic, the total energy necessary for writing becomes smaller. Reduction of the excess energy injection is expected to minimize the thermal interference. Furthermore, the low level of thermal diffusion in the heating and subsequent cooling process, as well as the negligible displacement of media during irradiation, makes the generated temperature profile steep and close to the original laser beam profile. This allows the mark size to be much less sensitive to the power fluctuation, which is particularly important when small marks are written without using the field modulation technique. We have already demonstrated in a static writing experiment and a numerical simulation [1] that written domain sizes closely fit the cross-sections of the laser power profile, and that the energy necessary for writing decreases considerably when the duration of laser irradiation is reduced to less than $10 \mathrm{~ns}$. The possibility of overwriting by using the through-thickness temperature gradient characteristic of shortpulsed irradiation has also been demonstrated $[2,3]$ The present paper describes how these features of short-pulsed writing are reflected in dynamic $\mathrm{read} /$ write experiments.

\section{EXPERIMENT}

A single track at $\mathrm{r}=30 \mathrm{~mm}$ of a commercially available 3.5-inch MO disk was used for all the experiments except for the amplitude measurement, where a disk with standard quadrilayer structure on top of unformatted (but grooved) glass substrate was used for the precise measurement. A high-speed laser driver was prepared and was combined with a conventional 830-nm laser diode. The efficiency of the optical head including the objective lens (N.A.= 0.55 ) was about $50 \%$. Typical light output waveforms of the write pulses are shown in Fig. 1. The rise/fall time $(10 \%-90 \%)$ of the light pulse increased from $0.6 \mathrm{~ns}$ to $1.1 \mathrm{~ns}$ with an increase in peak power. Because of the incomplete squareness of the pulse shape, the pulse energy was first obtained by directly integrating the light output waveform. The duration of the pulse was also measured in terms of the fullwidth half-maximum (FIVHM) of the light pulse. The peak power of the pulse was then defined as the energy divided by the measured pulse duration. The deviation of the measured duration (FIVHM) from 
the nominal width given by the pulse generator was less than $\pm 1 \%$ for nominal widths down to $5 \mathrm{~ns}$ for all the peak powers. But it increased to $-30 \%$ for a width of 1 ns at the highest peak power available. In the following, the nominal widths are used to indicate the degree of temporal localization of the injected energy. Both energy and power are expressed as quantities incident on the disk surface.

(a)

(b)

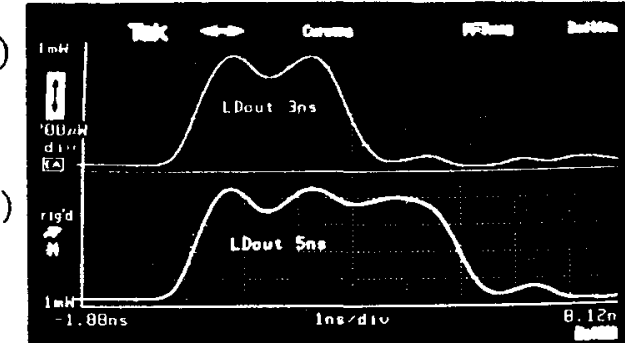

(c)

(d)

(e)

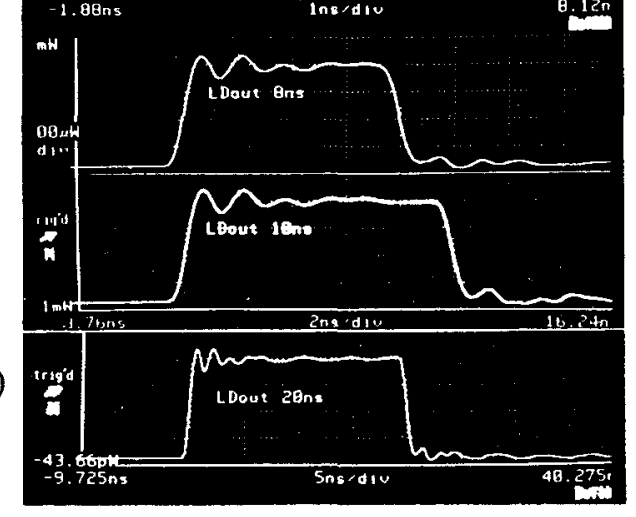

(f)

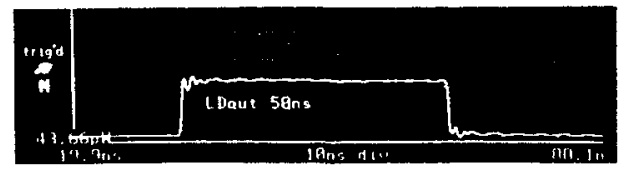

Fig. 1. Typical light output waveforms of write pulses. (a),(b) $1 \mathrm{~ns} / \mathrm{div}$, (c),(d) $2 \mathrm{~ns} / \mathrm{div}$, (e) $5 \mathrm{~ns} / \mathrm{div}$, and (f) $10 \mathrm{~ns} /$ div. Peak power is about $27 \mathrm{~mW}$ for (a) to (e), and $13 \mathrm{~mW}$ for (f) at the disk surface.

\section{RESULTS AND DISCUSSION}

For each duration, recording was done at various peak powers with the bottom (bias) power and the pulse repetition period fixed at $1.5 \mathrm{~mW}$ and $1 \mu \mathrm{s}$, respectively, and an energy-giving carrier level $2-3 \mathrm{~dB}$ higher than the noise level (hereafter called the write threshold energy) was measured. The write threshold energy is shown in Fig. 2 as a function of the pulse duration and as a function of the rotation speed (linear velocity). Owing to the very sharp transition in the power scan (as seen in Fig. 3), the write threshold energy is probably the most easily determined with minimum ambiguity, and is therefore used as a measure of the energy necessary for writing in the following discussion. For each rotation speed, the write threshold energy decreases almost linearly with decreasing pulse duration down to about 15 ns. The linear decrease in this range is ascribed to the constant rate of thermal diffusion and the movement of the spot during the laser irradiation. The decreasing rate of the write threshold energy becomes larger for pulse durations less than $12 \mathrm{~ns}$, indicating that the heating is approaching an adiabatic condition, that is, that the duration becomes comparable to the characteristic time of the thermal diffusion of the media. The write threshold energy for a duration of $3 \mathrm{~ns}$ at $1800 \mathrm{rpm}(5.65 \mathrm{~m} / \mathrm{s})$, for example, is only about $1 / 5$ of that for a duration of $70 \mathrm{~ns}$.

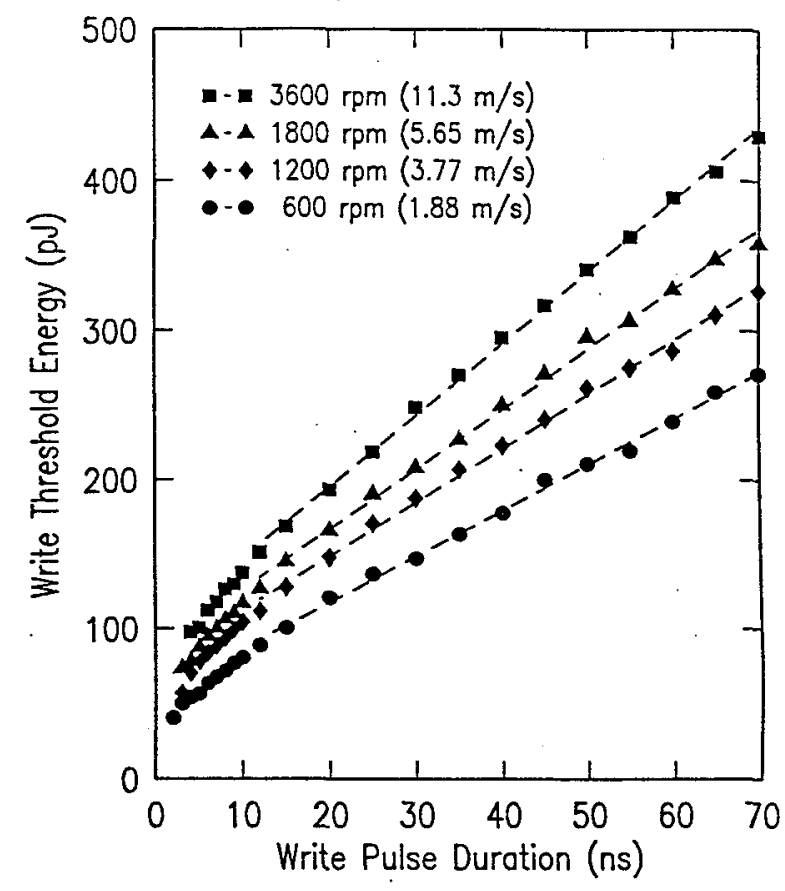

Fig. 2. Pulse duration dependence of the write threshold energy at various disk rotation speeds. $(r=30$ $\mathrm{mm}$. Write bottom power $=1.5 \mathrm{~mW}$. Write $/$ Erase field $=+1-500$ Oe.)

This write energy reduction is expected to produce the following results: (1) a steep temperature profile is generated, (2) the thermal interaction effect is minimized, (3) the linear velocity dependence of the optimum write power is minimized, and (4) the media degradation is minimized. The first two results will be discussed later in detail. As for the fourth result, we have not yet confirmed it experimentally, but it is a fairly straightforward consequence of the reduction in the total energy injection. The third result is discussed below. The improvement in the energy efficiency for writing is enhanced at high disk rotation speeds (as seen in Fig. 2), since the power density is significantly reduced by the movement of the spot during long irradiations. The write threshold energy, therefore, becomes less dependent on the rotation speed when the duration is reduced. In the 
short duration limit, the rotation speed dependence should disappear, but in fact it still remains, even for durations less than $5 \mathrm{~ns}$, as shown in Fig. 2. This is ascribed to the pre-heating effect of the fixed bottom power level, which has different influences at different rotation speeds: the efficiency of the pre-heating at the bottom power level decreases with increasing rotation speed. Since it is quite advantageous in terms of drive design that the optimum write power has little dependence on the linear velocity (which is particularly advantageous for drives with high data transfer rates), it is desirable to make the bottom (tracking) level as low as possible in order to take the best advantage of short-pulsed writing technique.

Figure 3 shows the carrier and noise level of the readout signal after recording with a pulse repetition period of $345 \mathrm{~ns}(2.9 \mathrm{MHH})$ and with pulse widths of 6,8 , and $70 \mathrm{~ns}$. For long-pulse recording ( $70 \mathrm{~ns}$ ), the increase in the noise level in the vicinity of the write threshold power is quite conspicuous, whereas it is small for short-pulsed recording (6 ns, $8 \mathrm{~ns}$ ). The suppression of the noise in this power range is not important from the practical point of view, but the reason for this phenomenon is of interest. For longpulse writing, the envelope of the temperature profiles throughout the irradiation and subsequent cooling process becomes gradual compared with that for short-pulsed writing, since a large amount of heat has to be injected to compensate for the loss due to thermal diffusion during irradiation. Because of the gradual profine; the domain size is quite sensitive to power fluctuation, particularly when small marks are written by using the very tip of the temperature profile (in other words, the low power close to the write threshold) [4]. Moreover, a fairly large area adjacent to the spot center experiences a temperature close to the Curie point, and this area is likely to produce micro-domains or mark edge irregularities, reflecting the write sensitivity fluctuation of the media. Small mark generation using low peak power has therefore been regarded as a not quite practical process. However, the observed noise suppression implies that short-pulsed writing may overcome this difficulty.

To see how short-pulsed writing suppresses the thermal interaction effect, we examined the readout signal amplitude, reducing the mark-to-mark distance. The recording was made at a radius of $r=30$ $\mathrm{mm}$ on an unformatted glass substrate disk rotating at $1800 \mathrm{rpm}$. The signal amplitude for conventional pulse-width (70 ns) recording and for the shortpulsed ( $6 \mathrm{~ns})$ recording are shown as functions of the write peak power in Fig. 4, where a pulse repetition period of $220 \mathrm{~ns}$ was used (the corresponding mark- to-mark distance was $1.24 \mu \mathrm{m}$, which is about $20 \%$ narrower than the shortest (3T) mark repetition period of the current-generation products). The maximum amplitude for 6-ns recording is larger than that for 70-ns recording in cases where the read path is common to both. Furthermore, the margin of the optimum write power (the power that gives the maximum amplitude in readout signal) is much wider in the short-pulsed case than in conventional writing. These results indicate that the thermal interference is certainly suppressed in short-pulse writing.

(a)

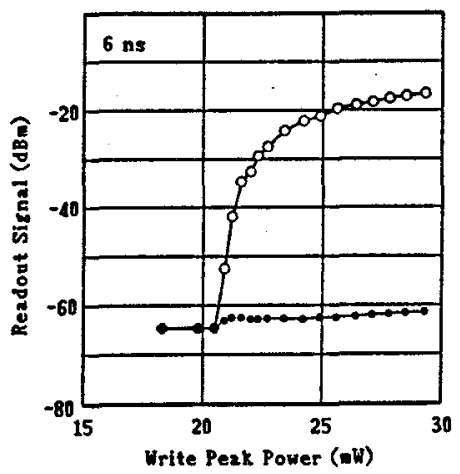

(b)

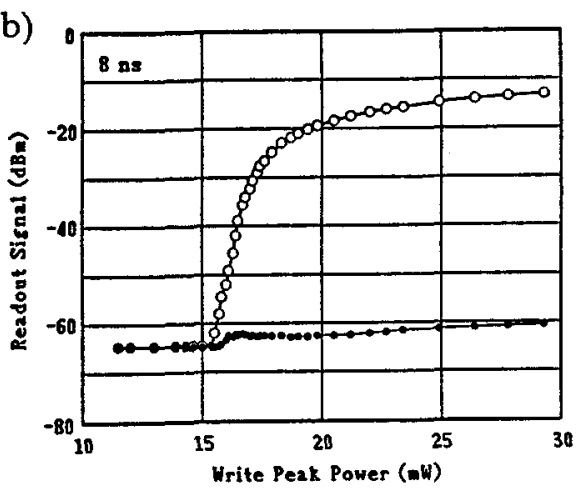

(c)

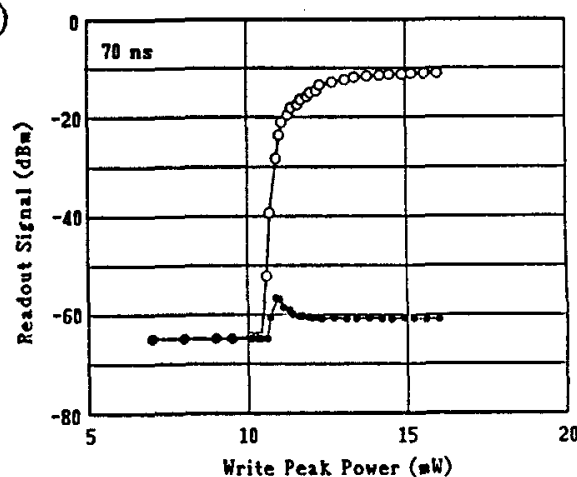

Fig. 3. The carrier and the noise level in the readout signal of the recording with a pulse repetition period of $345 \mathrm{~ns}(2.9 \mathrm{MHz})$ and with pulse widths of (a) 6, (b) 8 and (c) $70 \mathrm{~ns}$. (Position $\mathrm{r}=30 \mathrm{~mm}$, rotation speed $=1800 \mathrm{rpm}$, read power $=1.5 \mathrm{~mW}$, write bottom power $=0.5 \mathrm{~mW}$, write field $=300 \mathrm{Oe}$.) 


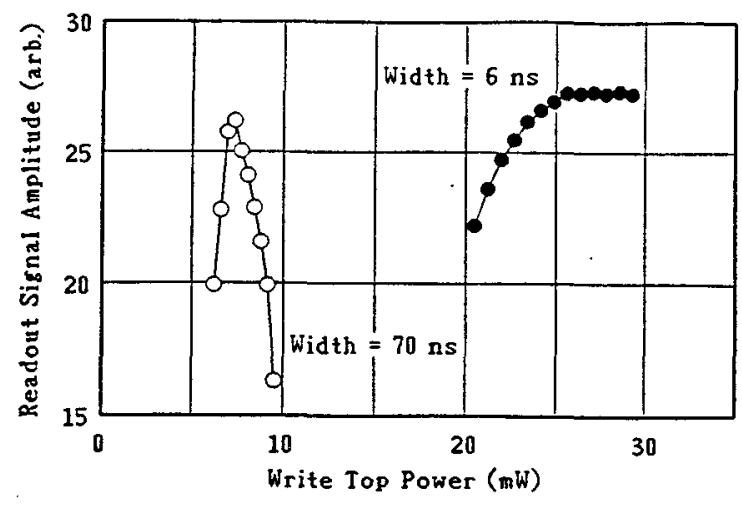

Fig. 4. The amplitude of the readout signal in shortpulsed recording (width $=6 \mathrm{~ns}$ ) and in conventional recording (70 ns) as a function of the write peak power. (Pulse repetition period $=220 \mathrm{~ns}$, write bottom power $=0.5 \mathrm{~mW}$, read power $=1.5 \mathrm{~mW}$, write field $=300 \mathrm{Oe}$.)

\section{CONCLUSION}

To achieve higher recording density in magneto-optical recording, a short-pulsed laser recording technique is proposed. By measurement of the energy necessary for writing, it was demonstrated that the write pulse duration of about $10 \mathrm{~ns}$ is comparable to the characteristic time for thermal diffusion in media. The writing energy for laser irradiation with a duration of less than $10 \mathrm{~ns}$ was found to be much smaller than that required in conventional writing. As a result, the thermal interaction within a mark or between marks is expected to be minimized. This was confirmed by writing the same dense patterns using short-pulsed irradiation and conventional irradiation, and by comparing the amplitude of their readout signals. It was also demonstrated that the low level of thermal diffusion associated with shortpulsed irradiation allows a steep temperature profile to be generated in media, so that the generation of small marks becomes much easier in short-pulsed writing than in conventional writing. Furthermore, this technique was found to have the potential to largely eliminate the linear velocity dependence of the optimum write power, by keeping write bottom (tracking) power level as low as possible. In addition, it is also important that the disk displacement during irradiation becomes negligible for short-pulsed irradiation, even for very high linear velocities.

\section{ACKNOWLEDGEMENTS}

The authors wish to thank $\mathrm{T}$. Ohtsuki and $\mathrm{S}$. Owa for their earlier contributions to this work, F. Sai and K. Kobayashi for helpful discussions, Y. Hirasaka for his help in optical head preparation, and
K. Noda and T. Suzuki for their help and encouragement.

\section{REFERENCES}

[1] T. Ohtsuki, S. Owa, and F. Yamada: Appl. Phys. Lett., 58, 2842 (1991).

[2] T. Ohtsuki, S. Owa, and F. Yamada: Appl. Phys. lett., 57, 105 (1990).

[3] T. Ohtsuki, C. J. Lin, and F. Yamada: IEEE Trans. Magn., 27, 5109 (1961).

[4] M. Takahashi, H. Sukeda, T. Nakao, T.Niihara, M. Ojima, and N. Ohta: Jpn. J. Appl. Phys., 28, Suppl. 28-3, 323 (1989) 